\title{
An Early North-Western Karaim Bible Translation from 1720. Part 1. The Torah
}

\author{
Michał Németh \\ Jagiellonian University in Krakow
}

\begin{abstract}
In this article an early Bible translation into north-western Karaim is described. The manuscript, written in Hebrew script (in its Karaim semi-cursive variant), dates back to 1720 , which makes it the oldest western Karaim Bible translation to be hitherto critically analysed. The manuscript was copied in Kukizów by Simcha ben Chananiel (who died in the 1720s). The language of the manuscript reveals archaic features, among others, consonant harmony in the process of emerging.
\end{abstract}

\section{Keywords}

western Karaim Bible translation, historical phonology of Karaim, vowel harmony, consonant harmony in north-western Karaim

\section{Preliminary remarks}

The present article presents a concise description of a manuscript that contains an early north-western Karaim Torah translation (so-called Chumash) from 1720, supplemented with a translation of the four books of Ketuvim. To my knowledge, this is the oldest translation of Bible fragments into Western Karaim hitherto described. ${ }^{1}$

1 For an overview of the existing editions of Karaim translations of Biblical texts, see OLACH (2013: 6-10) and JANKowski (2009: 504-509). 
The manuscript's language is not consistent. The Torah translation reveals archaic linguistic peculiarities, whereas the language of the books of Ketuvim is closer to present-day Karaim. For this reason, I decided to present the manuscript in two separate articles. In the present one, I will concentrate on the language of the Torah translation. Given the archaic quality of its language, and hence, the presence of a number of linguistic features that are fundamental as far as the history of north-western Karaim is concerned, a representative sample material will be presented below. I have chosen the parashah Yitro for this purpose. A portion of the second, most probably somewhat younger part, will be presented in a separate article as a continuation of the present one.

\section{The manuscript}

The manuscript contains the Five Books of Moses (fo. $1 \mathrm{r}^{\mathrm{o}}-341 \mathrm{r}^{\mathrm{0}}$ ) and four other books of the Ketuvim, namely the Book of Ruth $\left(342 \mathrm{r}^{\circ}-347 \mathrm{v}^{\circ}\right)$, the Book of Jeremiah $\left(348 \mathrm{r}^{\mathrm{o}}-358 \mathrm{r}^{\mathrm{r}}\right)$, Ecclesiastes $\left(358 \mathrm{v}^{0}-372 \mathrm{v}^{\circ}\right)$ and the Book of Esther $\left(373 \mathrm{r}^{0}-385 \mathrm{v}^{0}\right)$. It is stored in a private archive in Warsaw under the catalogue number III-73. Its owner wants to remain, for the time being, anonymous.

Thus, the manuscript consists of 385 folios with a size of $175 \times 140 \mathrm{~mm}$ and an approximate average of 21 lines per page. The original first two sheets are missing; folios $1 \mathrm{r}^{0}-2 \mathrm{v}^{0}$ contain a $19^{\text {th }}$-century addition in light brown ink written in south-western Karaim (the handwriting and the ink is typical for copyists from Halych; the copyist was most probably Jeshua-Josef Mordkowicz (1802-1884), but I can only say that based on the features of the handwriting). Folios 3-384 are copied in dark brown ink in north-western Karaim and originate from the $18^{\text {th }}$ century. The last fragment on folio 385 is in northwestern Karaim, again, but was added much later, apparently in the $20^{\text {th }}$ century, in black ink. I found the original folio 385 (containing Est. 9:28-10:3) severely damaged in a file stored in the same private archive (among a number of other different handwritten sheets), and have catalogued it under the number III-67-dok 3. The manuscript was subject to conservation in the latter half of the $20^{\text {th }}$ century. The text is clearly legible despite the ink corrosion visible on most folios. The text was fully vocalized at the same time the "main" text was written. ${ }^{2}$

2 KOWALSKI (1929: XIX, 289) observed that some non-vocalized manuscripts from Kukizów ended up in Halych after the community's fall in 1831 and received there a clearly 
The translation of the Torah was copied in the period between 25 March and 31 May $1720^{3}$ by Simcha ben Chananiel (died in the 1720 ; see KiziLov 2009: 53, 378), a known copyist of Hebrew manuscripts and the hazzan in Kukizów from 1709 until his demise. ${ }^{4}$ The date is expressed in the colophon that ends the Torah translation (on folios $340 \mathrm{v}^{0}-341 \mathrm{r}^{\circ}$ ):

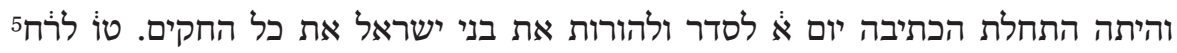

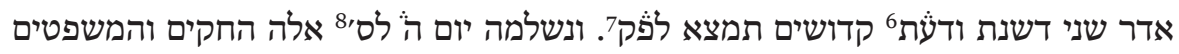

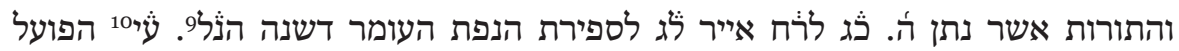

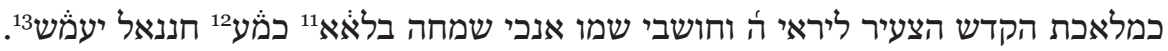

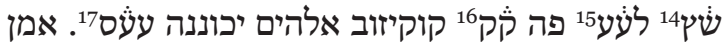

south-western type vocalization. This manuscript surely cannot be an example of such a practice even if we agree that the additions on folios 1-2 show that the manuscript must have ended up in the community of Halych.

3 I want to thank Prof. Piotr Muchowski (Poznań) for his invaluable help and suggestions in deciphering the date of the writing of the Torah translation. My thanks go also to prof. Tapani Harviainen (Helsinki) for his additional advices regarding the translation of the Hebrew colophon.

4 He was the second hazzan in this small community established in 1688 by six Karaim families, moving there from Trakai. The first to officiate there as hazzan was Mordechai ben Nisan (1688-1709).

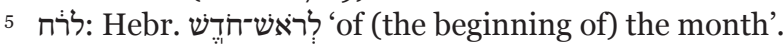

6 ודעודת: The first word of the quotation (see the translation below) indicates the date, i.e. letters waw + daleth + ayin + taw $=6+4+70+400=$ A.M. (5)480, i.e, A.D. 1720.

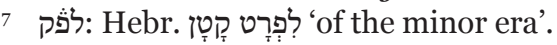

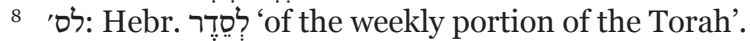

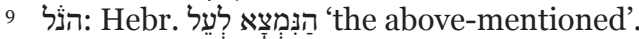

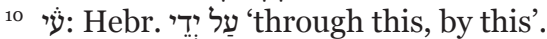

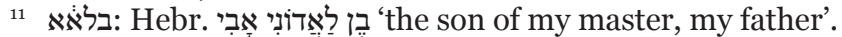

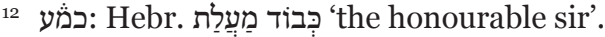

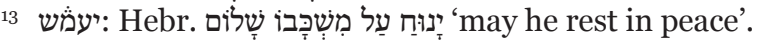

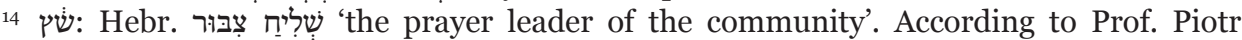
Muchowski (personal communication), this title could have referred to hazzans, too. This means that the use of this title does not clash with the fact that Simcha officiated as hazzan in Kukizów from 1709 until the 1720s.

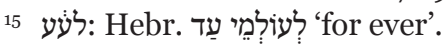

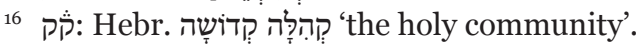

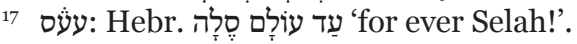


'And it started to be written on the first day of the seder Shemini, ${ }^{18}$ on the $15^{\text {th }}$ of Adar Sheni of the year $480^{19}$ of the minor era. And it was completed on the $5^{\text {th }}$ day of the seder Bechukotai, ${ }^{20}$ on the $23^{\text {rd }}$ of the month Iyar, on the $33^{\text {rd }}$ day of counting the Omer of the year mentioned above; by the performer of the holy enterprise is the young among the God-fearing and those who respect His name, by me, Simcha the son of my lord and my father, the honourable sir Chananiel may he rest in peace, the prayer leader of the community, for ever, here in the Holy Community of Kukizów may the Lord keep the community upright for ever Selah! Amen.'

This colophon is followed by the remaining 44 folios that contain the additional four books of Ketuvim mentioned above, which actually might suggest that these fragments were added somewhat later. However, there is no colophon or heading that would inform us when this happened.

A palaeographical examination clearly shows that the entire manuscript (except for the additions on the initial and the last folios) was copied by one person - Simcha ben Chananiel. This, in turn, suggests that the linguistic heterogeneity is a result of the copyist's use of different sources.

The manuscript was owned by several persons. On folio $341 \mathrm{r}^{0}$ Zecharia ben Shalom (died 1771?) made an annotation in Hebrew saying that he bought the book on the $28^{\text {th }}$ of Tevet 5528 , i.e. on 18 January 1768 from a person called Josef ben Jehuda. ${ }^{21}$ On folios $155 \mathrm{v}^{0}, 156 \mathrm{r}^{0}, 205 \mathrm{v}^{0}, 206 \mathrm{r}^{0}$, and $276 \mathrm{v}^{0}$ an oval seal has

18 The parashah is specified by a quotation read in this period, i.e. by a fragment from

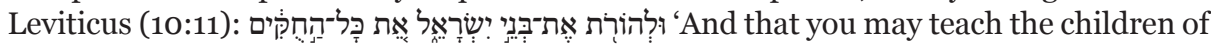
Israel all the statutes' (King James 2000).

19 The year number is expressed by ודלעת, i.e. the first word of a slightly altered quotation

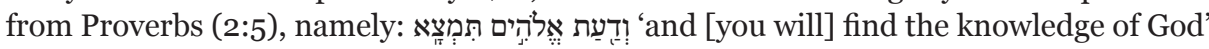

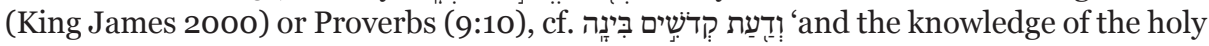
One is understanding' (King James 2000).

2o Again, this is specified by a quotation from Leviticus (26:46) read in the relevant period: These are the statutes and judgments and laws, which the Lord made' (King James 2000).

${ }^{21}$ Jehuda is mentioned as a deceased person with the abbreviation standing for Hebr.

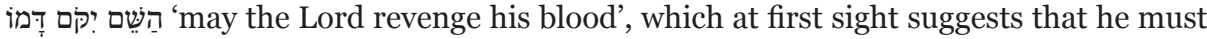
have been killed in Kotov (Pol. Kotów) or Derazhne (Pol. Derażne) during the Haidamak massacre in 1768. The book was, however, bought in January, whereas the Haidamak rebellion (the so-called Koliyivshchyna) broke out in spring of 1768. Ergo, Jehuda must have not necessarily been a citizen of Derazhne or Kotov. 
been pressed with the Hebrew inscription מרדכי בכר זכריה זל 'Mordechai the son of the honoured ribbi Zecharia of blessed memory'. On folio $205 \mathrm{~V}^{0}$ one of the owners listed the dates of birth of his three children (between 1789 and 1795). Finally, the original folio $385 \mathrm{v}^{\circ}$ (i.e. III-67-dok 3) contains the signature of a person called Shalom ben Zecharia (the signature is introduced with Hebr. אני 'I').22

\section{The transcription}

\subsection{Introductory remarks}

Since a number of phonetic features are hidden behind the Hebrew orthography and there are extremely modest subsidiary means that would help us establishing what was the actual phonetic value of the attested text, I could not use here a phonetic transcription, as I did so far in my editions of $19^{\text {th }}$-century Karaim texts.

The language of the analysed Torah translation clearly shows that the consonant-harmony we know from north-western Karaim was not fully developed in the time the manuscript was copied or translated..$^{23}$ In other words, the text originates from a transitional period when the harmony shift was still an ongoing process. Thus, in fact, such a transcription would be needed here that would cover two different sound systems (existing prior and after the

${ }^{22}$ Mordechai and Shalom were most probably brothers and were the sons of Zecharia ben Shalom who bought the manuscript in 1768 . The latter person, in turn, is most probably the same Zecharia ben Shalom to whom a kinah (lament for the dead) written the $13^{\text {th }}$ of Sivan 5531 A.M. (i.e. 26 May 1771) was devoted, written by Jeshua ben Mordechai Mordkowicz (chazzan in Halych until 1796). The latter information is to be found in manuscript JSul.I.37-14 stored in the archive of Anna Sulimowicz. I shall thank Anna Sulimowicz (Warsaw) for this invaluable suggestion.

${ }^{23}$ In north-western Karaim the front-back vowel harmony known, for instance, from other Middle Kipchak languages, was shifted towards palatal vs. non-palatal consonant harmony. I call this process harmony shift. To put it simply, from a synchronic point of view, we can say that this happened due to a process in which the palatal quality of the vowel has been shifted to the preceding consonant, namely due to the $\ddot{o}>{ }^{\prime} o, \ddot{u}>$ ' $u$ and $e>$ ' $a$ process (' indicates the palatality of the preceding consonant). Since this process could not have been described so far based on philological data in default of sources older than the late $18^{\text {th }}$ century, I have prepared a very detailed description of it and submitted it for publication in a separate, extensive paper (see NÉMETH 2014b). 
harmony shift), which means that a phonological transcription would not be satisfactory enough either. Ergo, the transcription I use must have remained partially conventional with a number of questions left open.

I have presented a detailed discussion of the matters related to transcription in NÉMETH (2014b). Below I summarize my reasoning.

\subsection{Open questions and the solutions chosen}

\subsubsection{Labial vowels: $\ddot{o}$, $\ddot{u}$ vs. 'o, 'u}

We do not know what was the actual phonetic value of the original front labials, i.e. $\ddot{o}$ and $\ddot{u}$ in non-initial positions in the time the manuscript was written. Eventually, word-medially and word-finally these vowels evolved into $o$ and $u$ with the preceding consonant being palatalized. ${ }^{24}$ But there were no orthographical means to distinguish between $\ddot{o}$ and 'o, or between $\ddot{u}$ and ' $u$ (the pairs were written with 〈i〉 and 〈i〉, respectively, with an additional aleph if written word-initially). Moreover, we cannot determine whether there was any $\ddot{o} \sim$ 'o and $\ddot{u} \sim$ ' $u$ alternation (similar to the widespread alternation of $e \sim$ ' $a$ described below) in the time the text was translated and copied. In the interests of clarity, I will use $\ddot{o}$ and $\ddot{u}$ in the transcription, in every position. The use of these symbols, however, should not be considered to be my "auto-da-fé" regarding their phonetic value; I do not think that the original ${ }^{*} \ddot{o}$ and $* \ddot{u}$ must have necessarily been pronounced as front labials in all positions in the time this Torah translation was performed, even if I consider this to be highly possible.

\subsubsection{The palatality of consonants}

It was only the position in front of ' $a$ that originated from ${ }^{*} e$ where the palatality of the preceding consonant was clearly noted by an additional yodh. ${ }^{25}$ Luckily for us, the orthography allows a clear distinction between $a$ and $e$, as well as between $a$ and ' $a$ : The sound $e$ was noted with the vowel points tzere () and (rarely) seghol (;) usually combined with the letter yodh, i.e. 〈»। and

${ }^{24}$ In word-initial position these vowels have remained $\ddot{o}$ and $\ddot{u}$ until the present day; $\ddot{o}$ did not occur in non-initial syllable, and therefore its use was limited to word-initial and word-medial positions.

25 The vowels $\ddot{o}$ or ' $o$ and $\ddot{u}$ or ' $u$ were also noted with the letter yodh (and waw), but we cannot determine whether it indicated the frontness of $\ddot{o}, \ddot{u}$ or the palatality of consonants in front of ' $O$ and ' $u$. 


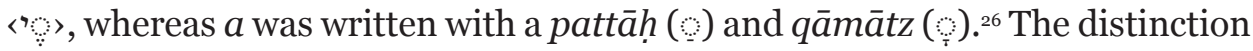
between $a$ and ' $a$, in turn, was marked with the letter yodh, i.e. the consonants

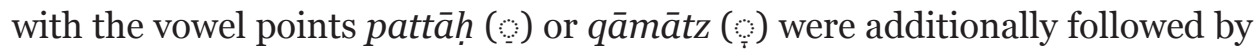
the letter yodh to denote ' $a$.

In front of front vowels ( $\ddot{o}, \ddot{u}, i$ and $e$ ) some of the consonants, for instance $k, g$ and $l$, were most probably palatalized in the time the analysed text was translated ${ }^{27}$ (the palatality of these consonants was not represented in writing). This feature, however, must have certainly been merely a phonetic one, and therefore I refrain from indicating this type of palatalization in the transcription. ${ }^{28}$

To sum up, in order not to use a transcription that would suggest far-fetching phonological or phonetic interpretation, I will note the palatality only of those consonants that stand in front of ' $a$ originating from ${ }^{*} e$. This solution seems reasonable for it does not obscure the difference between ' $a$ and $a$ regularly represented in the writing.

${ }^{26}$ Word-initially aleph was used as mater lectionis. Word-finally the notation of both $a$ and $e$

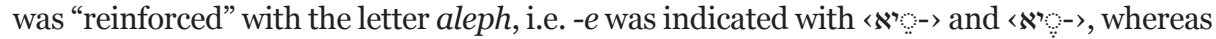

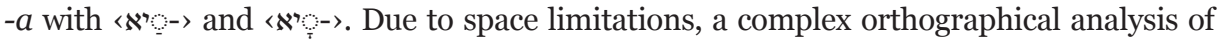
the text cannot be performed here. This, however, definitely deserves a separate study; preferably as a complementary description to a complete critical edition of the text.

27 The palatal pronunciation of $k, g$ and $l$ is a well-known feature of Turkic phonotactics. Importantly, this feature is present in both dialects of Modern Western Karaim (see e.g. KOWALSKI 1929: XLVII; ZAJĄCZKOWSKI 1931: 9), which allows us to presume that it is rather an inherited feature. Cf. our remarks in 5.1.

${ }^{28}$ In fact, according to one of the acceptable and, in fact, highly probable scenarios modelling step by step the evolution of the harmony shift it may well be that it was the consonants that became palatalized first as a result of the already existing process of palatalization of $k, g$ and $l$ before front vowels, combined with the strong influence of Lithuanian and Slavonic (above all Polish and Russian) phonotactics, leading to an increase in the number of palatalized consonants preceding front vowels which, in turn, weakened the opposition between front and back vowels and made the backing of front vowels possible. According to this scenario, the front vowels were backed later, as a next step, starting with word-final syllables (see below), and this process gradually expanded towards the beginning of words without coming to an end: we know from present-day Karaim that $e$ remained unchanged in the first syllable, whereas $\ddot{o}$ and $\ddot{u}$ remained untouched in word initial position (for the overall model, see NÉMETH 2014b). The hypothetical palatality of these consonants in the transitional period (i.e. after the strong palatalization process of consonants, but before the process of backing the front vowels) should, however, also be treated as a phonetic rather than phonological feature. 


\subsubsection{The phonetic value of $q$}

Another question is what was the actual pronunciation of the sound represented by the letter koph (ק) syllable-finally and suffix-initially? Eventually, it evolved into $[\chi]$ in these positions, but we do not know when this happened. In this respect, the spelling of north-western Karaim texts written or printed in Hebrew script could have been etymological, at least until the second half of the $19^{\text {th }}$ century. ${ }^{29}$ I will use $q$ for the original ${ }^{*} q$ in every position.

\subsubsection{The value of the letter yodh in $1^{\text {st }}$ and $2^{\text {nd }}$ pl. person markers}

In the Hebrew script no distinction can be made between the back unrounded vowel $y$ and its front counterpart $i$. For this reason, it remains an open question what was the phonetic value of the letter yodh in the $1^{\text {st }}$ and $2^{\text {nd }} \mathrm{pl}$. person markers written as ביזיז-> into -byz $\sim$-biz > -byz -biź and -syz -siz > -syz -śiź, but we cannot determine when this process exactly took place. In the transcription I use the original forms -biz and -siz. Cf. also 5.3 below.

\section{The sample linguistic material}

\subsection{Introductory remarks}

Below I present the transcription of the parashah Yitro. My English translation follows as close as possible the Karaim original and is based on the King James Bible 2000 and on the English Standard Version. In the footnotes, I also provide a brief comparison with another translation of the Torah, written in south-western Karaim with some archaic linguistic features being preserved, copied in the $19^{\text {th }}$ century by Jeshua-Josef Mordkowicz (catalogue

29 Manuscripts from this period already show the letter cheth $\langle\Pi\rangle$ and koph with a rafe,

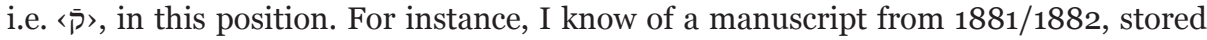
under catalogue number III-68 in the same collection, which was written by the 14-yearold Simon Osipowicz Chorczenko (born 1868), in which the young author applied an orthography based on the actual pronunciation rather than philological tradition. For קוּלְוֹח

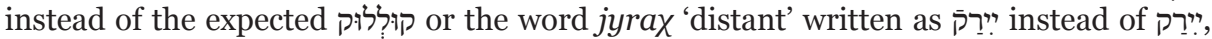
which informs us that the $-k>-\chi$ change must have happened prior to the 1880 .

3o Cf. SWKar. -biz and -siz, Kipch. -biz and -siz (von GABAIN 1959: 64). 
number JSul.III.01; folios $77 \mathrm{v}^{0}-79 \mathrm{v}^{\circ}$ ) and stored in another private archive in Warsaw. ${ }^{31}$ The English translation, however, corresponds to the northwestern Karaim text in manuscript III-73 only. In this comparison, I will ignore differences that are merely phonetic in nature. If a comment concerns not a word, but a longer fragment, the text in question will be enclosed in half square brackets, i.e. L...?

\subsection{Transcription}

\section{Exodus 18:1-27}

Page $113 \mathrm{~V}^{\mathrm{o}}$

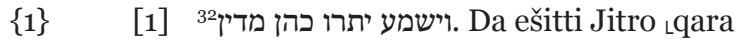
\{2\} [2] tonlusu ${ }^{733}$ Midjannyn qajnatasy Mošenin ošol bar-
\{3\} [3] ča ne ${ }^{34}$ ki qyldy tenri Mošege da Israelge ulusuna „özü-
\{4\} [4] nün ${ }^{735}$ ki čygardy Adonaj3 ${ }^{66}$ ošol Israelni Micrid’an. ויקח. Da
\{5\} [5] aldy Jitro qajnatasy Mošenin ošol Ciporany qatynyn Mošenin
\{6\} [6] uzatqanyndan sortun any.
\{7\} [7] lary anyn ki aty ol birisinin ${ }^{38}$ Geršom ki ajtty garib boldum ${ }^{39}$
\{8\} [8] jat jerda. Da aty ol birisin ${ }^{40}$ Eliezer ajtadog I $^{4} \check{c}^{41}$
\{9\} [9] ki tenrisi atamnyn boldu ${ }^{42}$ bolušlugumda ${ }^{43}$ da qutqardy
\{10\} [10] meni qylyčyndan paronun. ויבוא. Da keldi Jitro qajnatasy

${ }^{31}$ My aim is not to perform a comparative critical edition but only to present the text of the manuscript from 1720 in a larger comparative perspective.

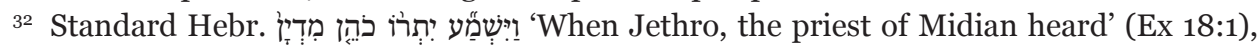
King James 2000.

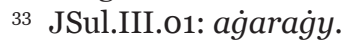

34 JSul.III.o1: neni.

35 JSul.III.01: this word is absent.

36 JSul.III.o1: $H$, i.e. ה. I will not indicate this difference any more.

37 JSul.III.o1: additionally: ot qonarlyqtan necik baryredi qajtma Micrige.

${ }^{38}$ JSul.III.01: birinin.

39 JSul.III.o1: edim

40 Probably a clerical error, see my remark in the translation. JSul.III.01: ekincinin.

41 JSul.III.01: this word is absent.

42 JSul.III.01: edi.

43 JSul.III.o1: boluslugiumda menim.

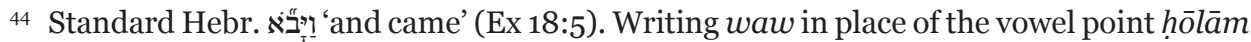
was a common practice among Karaim copyists, also in translations into Eastern Karaim. 
\{11\} [11] Mošenin da uvullary anyn ${ }^{45}$ da qatyny anyn ${ }^{46}$ Mošege ol midbaraga

\{12\} ki ol toxtaredi anda tavyna ol tenrinin. ויאמר. Da

\{13\} [13] ajtty Jitro „elči ašyra ${ }^{147}$ Mošege men qajnataj ${ }^{48}$ Jitro kelemen

\{14\} saja da qatynyj ${ }^{49}$ da eki uvullary anyn birgesińa ${ }^{50}$. ויצא.

\{15\} [15] Da čyqty Moše uturusuna qajnatasynyn da bašurdu da

\{16\} [16] öptü any da sordular kiši dostundan savluqnu ${ }^{51}$ da kel-

\{17\} [17] diler ol čatyrg்a. ויספר. Da qotardy Moše qajnatasy-

\{18\} [18] na ošol barča ne ${ }^{52}$ ki qylady Adonaj paroġa da Micriǵa

\{19\} [19] ¿Israel üčün ${ }^{753}$ ošol bar ol jadavny ${ }^{54}$ ki učrady alarny jolda kij ${ }^{55}$

\{20\} [20] ¿uruštu alar byla amalek ${ }^{56757}$ da qutqardy alarny Adonaj. ויחד.

\{21\} [21] Da bijendi Jitro bar ol jaxšylyq ${ }^{58}$ üčün ki qyldy Adonaj Israelge

\{22\} [22] \{\{catchwords:\}\} ki qutqardy

\section{Page $114 \mathrm{r}^{0}$}

\{1] ki qutqardy any qolundan Micrinin. ויאמר. Da ajtty

\{24\} [2] Jitro maxtavludu ${ }^{59}$ Adonaj ki qutqardy sizni qolundan Micrinin da

$\{25\} \quad[3]$ qolundan paronun $k^{60}$ qutqardy ošol ol ulusnu erkitü-

\{26\} [4] vünd'an Micrinin. Hתה. Haligine bildim ${ }^{61}$ ki ulluraqty $^{62}$

45 JSul.III.01: this word is absent.

46 JSul.III.o1: Mošenin.

47 JSul.III.o1: this fragment is absent.

48 JSul.III.01: qajnatan senin.

49 JSul.III.01: qatynyn senin.

5o JSul.III.o1: birgesine anyn.

${ }^{51}$ JSul.III.o1: bazlyqny.

${ }^{2}$ JSul.III.o1: neni.

53 JSul.III.o1:isleriücünIsraelnin.TheTorah translation copied by Jeshua-Josef Mordkowicz I use for comparison reflects traces of the transitional period in south-western Karaim when the $\ddot{o}>e, \ddot{u}>i$ delabialization process was operating and $\ddot{o} \sim e$ and $\ddot{u} \sim i$ were alternating. This is why we have ücün (and not icin) here. This process took place most probably in the last decades of the $18^{\text {th }}$ century, somewhat later than the $\breve{s}>s$ change. For a detailed analysis of these changes based on philological data, see NÉmETH (2014a).

54 JSul.III.o1: ucurnu.

55 JSul.III.o1: da ki.

${ }^{6}$ Hebr. עִמָלִ 'a wicked person'.

57 JSul.III.01: this fragment is absent.

${ }^{8}$ JSul.III.01: jaxsy.

59 JSul.III.01: maxtavludur.

6o JSul.III.o1: ki qutqardy sizni qolundan Micrinin da qolundan paronun ki.

${ }^{61}$ JSul.III.01: bilemen.

62 JSul.III.01: ulluraqtyr. 
\{27\} [5] Adonaj bar ol malaqlardan ${ }^{63}$ ki nendij iš byla ki čajalyq etti-

\{28\} [6] ler alaj ${ }^{64}$ qajtardy tölev ${ }^{65}$ alarga. Da aldy Jitro

\{29\} [7] qajnatasy Mošenin ola ${ }^{66}$ da debexalar tenriǵa da keldi Aharon

\{30\} [8] da bar qartlary Israelnin ašama ötmek qajnatasy byla Moše-

\{31\} [9] nin alnynda ol tenrinin. Da edi tanbyladan da

\{32\} [10] olturdu Moše töre etḿa ošol ol ulusnu da tur-

\{33\} [11] du ol ulus Lalnynda Mošenin ${ }^{767}$ ol tandan ${ }^{68}$ ol ingirgedejin.

\{34\} [12] Da kördü qajnatasy Mošenin ošol barča ne ${ }^{69}$ ki ol

\{35\} [13] qyldy ulusqa da ajtty nedir ${ }^{70}$ ol iš ošpu ${ }^{71}$ ki sen qyla-

\{36\} [14] sen ulusqa ne üčün sen olturasen jalgyz özüj Łtöre

$\{37\} \quad[15]$ etme ${ }^{72}$ da bar ol ulus Łköplügündan törelernin turady-

\{38\} [16] lar alnyjda tandan ingirgedejin773. ויאמר. Da ajtty

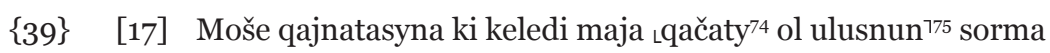

\{40\} [18] sözün tenrinin. כי Alajoq ${ }^{76}$ ki bolsa alarga töre sö-

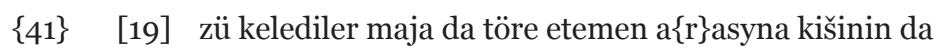

$\{42\}[20]$ arasyna dostunun ${ }_{\llcorner}$da ol soruvčularga sözün tenrinin 777 da

\{43\} [21] bildiremen alarğ $a^{78}$ ošol resimlerin ol tenrinin da ošol

\section{Page $114 \mathrm{~V}^{\mathrm{o}}$}

\{44\} [1] üvretüvlerin anyn. ויאמר. Da ajtty qajnatasy Moše-

\{45\} [2] nin anar jaxšy tüvüldü ol iš ki sen qylasen. נבל [2]. Upran-

63 JSul.III.o1: tenrilerden.

${ }^{64}$ JSul.III.01: Micrililer.

65 JSul.III.o1: this word is absent.

${ }^{66}$ Hebr. עוֹ עi 'burnt offering'.

${ }_{67}$ JSul.III.01: Moše qatyna.

68 JSul.III.01: erten byladan.

69 JSul.III.01: neni.

70 Sul.III.01: nedi.

${ }^{71}$ JSul.III.o1: ol ospu.

${ }^{72}$ JSul.III.o1: this fragment is absent.

73 JSul.III.o1: turady senin katyna baslap erteden ingirgedejin.

74 Perhaps < Hebr. קְָּ 'part, a small part'.

75 JSul.III.01: ol ulus.

${ }^{76}$ JSul.III.01: this word is absent

77 JSul.III.o1: this fragment is absent.

${ }^{78}$ JSul.III.o1: this word is absent. 
\{46\} [3] ma upranyrsen dağyn ol79 ulus ošpu ${ }^{80} \mathrm{ki} \mathrm{birgeje}^{81} \mathrm{ki}$

\{47\} [4] avurdu sendan ${ }^{82}$ ol iš bolalmassen qylma any jalg̀yz özüj.

\{48\} [5] Haligine tynlaġyn sözüme keneš berejim saja da

\{49\} [6] bolsun bolušluggu tenrinin birgeje ${ }^{83}$ bolġun sen Łulus üčün al-

$\{50\} \quad[7]$ nynda ol tenrinini ${ }^{84}$ da keltirgin sen ošol ol sözlerni ${ }^{85}$ alny-

\{51\} [8] na ol tenrinin. והזהרתה. Da zynharlaġyn alarġa ošol

\{52\} [9] ol resimlerni da ošol ol üvretüvlerni da bildirgin alar-

\{53\} [10] g ga ošol ol jolnu ki barğajlar ${ }^{86}$ anyn byla da ošol ol išni

\{54\} [11] ki qylgajlar. ואתה. Da sen baqqyn bar ol ulustan tuvušlu

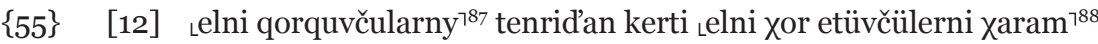

\{56\} [13] malny da qojgun alar üstüne agalyqlaryn ${ }^{89}$ minlernin aġalyq-

\{57\} [14] \{laryn $\}^{90}$ jüzlernin aġalyqlaryn ${ }^{91}$ enlilernin da agalyqlaryn ${ }^{92}$ onlarnyn.

\{58\} [15] ושפטו. Da töre etsinler93 ošol ol ulusnu har

\{59\} [16] vaxtta ${ }^{94}$ da bolgaj bar ol ullu Łišni keltirsinler ${ }^{795}$ saja

$\{60\}$ [17] da bar ol kiči Lišni töre etsinler ${ }^{196}$ özleri da jengillet-

\{61\} [18] kin ${ }^{97}$ üstüjdan ${ }^{98}$ da Łkötürsünler birgeja ${ }^{799}$. אם. Eger

\{62\} [19] Lišini ošpunu ${ }^{100}$ qylsaj da bujursa saja tenri da bolalyr-

\footnotetext{
79 JSul.III.o1: sen dag்yn ol.

80 JSul.III.o1: ol ospu.

81 JSul.III.o1: birgene senin.

82 JSul.III.01: senin $\{i c i n\}$.

${ }_{3}$ JSul.III.o1: birgene senin.

84 JSul.III.o1: ulusqa ivretivci saruvlaryn tenrinin.

85 JSul.III.o1: sözlerni ki sorsalar senden.

86 JSul.III.o1: jirigejler.

87 JSul.III.01: el qorquvcular.

88 JSul.III.o1: el Xor etivciler qyng்yrlyq.

89 JSul.III.01: ag்araqlaryn.

90 JSul.III.01: ag்araqlaryn.

91 JSul.III.01: ag்araqlaryn.

92 JSul.III.01: ag்araqlaryn.

93 JSul.III.o1: eterler.

94 JSul.III.o1: vaxt.

95 JSul.III.o1: sözni keltirirler.

96 JSul.III.o1: sözni tere eterler.

97 JSul.III.01: jengil etkin.

98 JSul.III.o1: özün istinden.

99 JSul.III.01: ketirirler birgene senin.

${ }^{100}$ JSul.III.o1: osol ol isni ol uspu.
} 
\{63\} [20] sen turma da daġyn bar ol ulus ošpu ${ }^{101}$ ornuna kelir

\{64\} [21] Lbazlyq byla ${ }^{1102}$. וישמע. Da tynlady Moše sözüne qajnatasyna

\{65\} [22] \{\{catchwords: $\}\}$ da qyldy

\section{Page $115 \mathrm{r}^{\mathrm{o}}$}

\{66\} [1] da qyldy barča ne ${ }^{103}$ ki ajtty. Da sajlady Moše tuvuš-

\{67\} [2] lu elni ${ }^{104}$ bar Israelden da berdi alarny agaraq ${ }^{105}$ ol ulus üs-

$\{68\} \quad[3]$ tüne agalyqlaryn ${ }^{106}$ minlernin agalyqlaryn ${ }^{107}$ jüzlernin agalyqlaryn ${ }^{108}$ en-

\{69\} [4] lilernin da aġalyqlaryn ${ }^{109}$ onlarnyn. Da töre ete-

\{70\} [5] rediler ošol ol ulusnu har vaxtta ${ }^{110}$ ošol ol qaty söznü

\{71\} [6] keltirirediler Mošege da bar ol kiči nerseni ${ }^{111}$ töre e-

\{72\} [7] terediler özleri. וישלח. Da uzatty ${ }^{112}$ Moše ošol

\{73\} [8] qajnatasyn da bardy özüne jerińa ...

\section{Exodus 19:1-25}

\{74\} [9] čü jangajda ${ }^{113}$ čyqmagyna Łulanlarynyn Israel ${ }^{114}$ jerindan Micrinin

\{75\} [10] ošpu ${ }^{115}$ künde keldiler midbaryna Synaj\{nyn\}. ויסעו. Da

\{76\} [11] köčtüler Refidimden da keldiler midbaryna Synajnyn da

\{77\} [12] toxtadylar midbarda da toxtady anda Israel qaršysyna

\{78\} [13] ol tavnyn. Da Moše mindi alnyna ol tenri-

\{79\} [14] nin da čaġyrdy anar Adonaj ol tavdan ajtadoġač bulaj ajt-

\footnotetext{
${ }^{101}$ JSul.III.o1: ol uspu.

${ }^{102}$ JSul.III.o1: bazlyqta.

103 JSul.III.o1: neni.

104 JSul.III.01: el.

${ }^{105}$ JSul.III.01: ag $\operatorname{saraqlar.}$

${ }^{106}$ JSul.III.o1: ag araqlaryn.

${ }^{107}$ JSul.III.o1: ag்araqlaryn.

${ }^{108}$ JSul.III.o1: ag்araqlaryn.

${ }^{109}$ JSul.III.01: ajaraqlaryn.

${ }^{110}$ JSul.III.01: vaxt.

${ }^{111}$ JSul.III.o1: sözni.

${ }^{112}$ JSul.III.o1: $\dddot{j} d i$.

${ }^{113}$ JSul.III.o1: ajda.

${ }^{114}$ JSul.III.01: uvullarynyn Israelnin.

${ }^{115}$ JSul.III.o1: ol ospu.
} 
\{80\} [15] qyn üvüne Jaaqovnun da Łanlatqyn ulanlaryna ${ }^{7116}$ Israelnin.

\{81\} [16] אתם. Siz kördüjüz ošol ne ${ }^{117}$ ki qyldym Micrige da

\{82\} [17] Łkötürdüm sizni qyjasa nešer qanatlary üstüne ${ }^{118}$ da

\{83\} [18] keltirdim sizni özüme. Da haligine eger

\{84\} [19] tynlama tynlasajyz ünüme da saqlasajyz ošol šertimni

\{85\} [20] da bolursiz maja onča bar ol uluslardan ki menimdi

\{86\} [21] bar ol jer. ואתם. Da siz bolursiz maja bijligi kohen-

\section{Page $115 \mathrm{~V}^{0}$}

\{87\} [1] lernin ${ }^{119}$ da aziz xanlyq bulardylar ošol ${ }^{120}$ sözler ki Łsözlegin ulanlary-

\{88\} [2] na na keldi Moše da ündedi ${ }^{123}$ qartlaryn ${ }^{124}$

\{89\} [3] ol ulusnun da qojdu alynlarynda alarnyn ošol bar ol söz-

\{90\} [4] lerin ošpularny ki LSarydy any ašyra ${ }^{125}$ Adonaj. Da

\{91\} [5] qaruv berdiler bar ol ulus \{birge $\}^{126}$ da ajttylar barča ne ${ }^{127}$ ki sözledi

\{92\} [6] Adonaj qylarbiz da qaruv qajtardy Moše ošol sözlerin ol

\{93\} [7] ulusnun Adonajgia. ויאמר. Da ajtty Adonaj Moše-

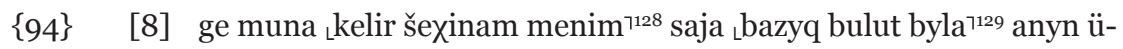

\{95\} [9] čün ki $^{130}$ ešitkej ol ulus sözlegenimda birgeje ${ }^{131}$ da dağyn

\{96\} [10] Lsaja inamlyq bergejler ${ }^{132}$ dunjaġa dejin da anlatty Moše ošol

\footnotetext{
${ }^{116}$ JSul.III.01: sözlegin anlatadoġac uvullaryna.

117 JSul.III.o1: neni.

118 JSul.III.o1: elttim sizni qanatlary istine nešerlernin.

${ }^{119}$ JSul.III.01: agaraqlarnyn.

${ }^{120}$ JSul.III.o1: ol.

${ }^{121}$ JSul.III.o1: sözlegejsen uvullaryna.

${ }^{122}$ Standard Hebr. וָיביבא 'and came' (Ex 19:7).

${ }^{123}$ JSul.III.o1: cag்yrdy.

${ }^{124}$ JSul.III.01: qartlaryna.

${ }^{125}$ JSul.III.o1: bujurdu anar.

${ }^{126}$ JSul.III.01: birde.

127 JSul.III.o1: neni.

${ }^{128}$ JSul.III.o1: men kelimen sana; the form kelimen comes from kelirmen. For examples of eliminating the future tense marker in south-western Karaim non-abbreviated forms see NémETH (2011a: 47). So far, this type of syncopation was thought to be characteristic only of secular texts reflecting the every-day language.

129 JSul.III.01: qalynlygy byla ol bulutnun.

${ }^{130}$ JSul.III.01: this word is absent.

${ }^{131}$ JSul.III.o1: birgene senin.

${ }^{132}$ JSul.III.o1: naviligine senin inangajlar.
} 
\{97\} [11] sözlerin ol ulusnun adonajgia. ויאמר. Da ajtty

\{98\} [12] Adonaj Mošege barg்yn ol ulusqa da aziz ${ }^{133}$ etkin alarny

\{99\} [13] bügün da tanbyla da juvsunlar upraqlaryn özlerinin ${ }^{134}$.

\{100\} [14] והיו. Da bolsunlar hadirler ol üčünčü künge ki

\{101\} [15] ol üčünčü künde ener šexinasy Adonajnyn közleriče

\{102\} [16] bar ol ulusnun tavy üstüne Synajnyn. והגבלת.

\{103\} [17] Da čeklegin ošol ol ulusnu čüvre ajtadogač saqlanyjyz

\{104\} [18] özüjüzge minmekten tavġa da tijmekten učuna anyn

\{105\} [19] bar ol tijüvčü tavġa ölme öltürülsün. לא.

\{106\} [20] ¿Da kim ki čyqsa ol čektán tijmesin anar qol ki ančaq

\{107\} [21] eger juvuq bolsa ol tašbyla tašlanma tašlansyn a

$\{108\}$ [22] \{\{catchword:\}\} eger

\section{Page $116 \mathrm{r}^{\circ}$}

\{109\} [1] eger jyraq bolsa oqbyla oqlanma oqlansyn hem kiši

\{110\} [2] hem tuvar tiri bolmasyn tartqanda ol šofar ${ }^{135}$ išlengen

\{111\} [3] qočqar müvüzündan ol vaxtta barsynlar čatyrlaryndan tur-

\{112\} [4] ma ol tav tübüne ${ }^{136}$. וירד. Da endi Moše ol tavdan

\{113\} [5] ol ulusqa da azizizi etti ošol ol ulusnu da juvdular

\{114\} [6] upraqlaryn özlerinin ${ }^{138}$. ויאמר. Da ajtty ol ulusqa

$\{115\} \quad[7]$ bolujuz hadirler üč künlerǵa juvumajyz qatynġa.

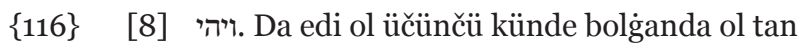

\{117\} [9] da boldu avazlar da jyldyrymlar da ${ }_{\llcorner}$küčlü bulut ${ }^{139}$ ol tav üs-

\{118\} [10] tüne da šofar avazy küčlü astry da qaltrady bar ol

\{119\} [11] ulus ki avulda. Da čyġardy Moše ošol ol ulus-

$\{120\} \quad[12]$ nu alnyna ${ }^{140}$ ol tenrinin ol avuldan da turdular Łtübünde

${ }^{133}$ JSul.III.01: ajryqsy.

134 JSul.III.01: this word is absent.

135 < Hebr. שוֹפָר 'trumpet'.

${ }^{136}$ JSul.III.o1: different wording in the entire verse: Da bulaj eltirilsin ol cyg்uvcu cekten tijmesin anar qol ancaq tas byla taslansyn juvuq bolsa a eger jyraq bolsa oq byla atylma atylsyn kle tuvar kle kisi tiri bolmasyn tartqanda ol joel šofary alar erkli bolurlar minme tavga.

${ }^{137}$ JSul.III.01: ajryqsy.

${ }^{138}$ JSul.III.01: this word is absent.

${ }_{139}$ JSul.III.o1: bulut küclü.

${ }^{140}$ JSul.III.o1: utrusuna. 
\{121\} [13] ol tavnyn ${ }^{141}$. והר. Da tavy Synaj\{nyn\} tütünlendi ${ }^{142}$ barčasy $^{143}$ anyn

\{122\} [14] üčün ki endi anyn üstüne šexinasy Adonajnyn ot-

\{123\} [15] byla da kötürüldü Łtütünü kibik ${ }^{144}$ ol kireč pečinin da

\{124\} [16] qaltyrady bar ol tav astry. Da edi Łol šofar

\{125\} [17] avazy ${ }^{1145}$ baryredi da küčejiredi astry Moše sözleredi

\{126\} [18] da ol tenri _arttyryredi ${ }^{146}$ anar ${ }_{\llcorner}$küč avazynda ki ešit-

\{127\} [19] kejler ulanlary Israelnin sözlerin Mošenin tartmaq vaxt-

\{128\} [20] ta ol šofar ${ }^{7147}$. וירד. Da endi šexinasy Adonajnyn tavy

$\{129\}$ [21] üstüne Synajnyn bašyna ol tavnyn da ündedi ${ }^{148}$ Adonaj

\section{Page $116 \mathrm{v}^{\mathrm{o}}$}

\{130\} [1] Mošeni ${ }^{149}$ bašyna ol tavnyn da mindi Moše. ויאמר. Da

\{131\} [2] ajtty Adonaj Mošege engin tanyq etkin ulusta maġat

\{132\} [3] ¿buzarlar ol čekni alnynda Adonajnyn baqma ${ }^{150}$ da tüšer andan

\{133\} [4] köbüsü. Da dagyn ol kohenler ol juvuvčular Lalny-

\{134\} [5] na Adonajnyn azizlensinlar ${ }^{151}$ magat buzuqluq qylar alarda Adonaj.

\{135\} [6] Da ajtty Moše Adonajg̈a bolalmasty ol ulus

\{136\} [7] minme tavyna Synajnyn ki sen tanyq ettij bizde ajtadoġač

\{137\} [8 c čeklegin ol ${ }^{152}$ tavny da aziz etkin any. Da ajt-

\{138\} [9] ty ${ }^{153}$ Adonaj anar barg்yn engin da minersen ${ }^{154}$ sen da Aharon Łbirgeje

\{139\} [10] ol vaxtta ki necik bujursam sizge da ol kohenler da

\{140\} [11] ol ulus buzmasynlar čekni ${ }^{155}$ minme alnyna Adonajnyn magat

\{141\} [12] buzuqluq qylar alarda. וירד. Da endi Moše ol ulus-

$\{142\} \quad[13]$ qa da ajtty alarga ...

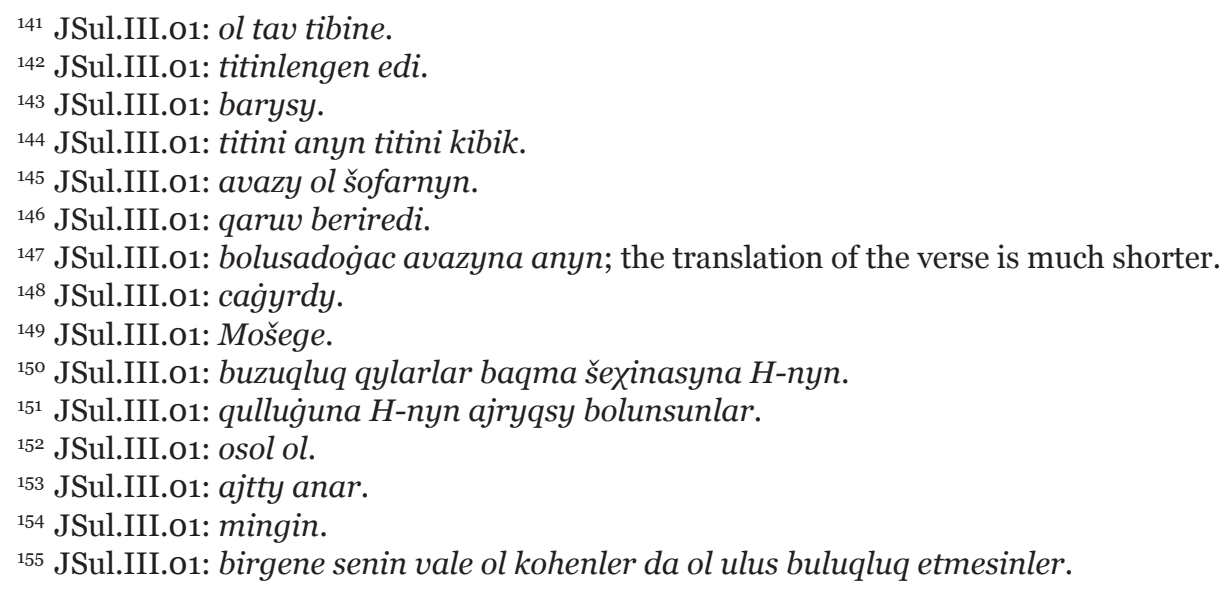




\section{Exodus 20:1-23}

$\{142\} \quad[13]$

.... Da sözledi tenri ošol

\{143\} [14] bar ol sözlerni ${ }^{156}$ ošpularny ajtadoġač. Mendir

\{144\} [15] men Adonaj tenrij senin ki čygardym seni jerindan Micrinin qullar

\{145\} [16] erkinden. Bolmasyn saja özge tenriler menim

\{146\} [17] qajamlygymm alnyna. לא. Qylmaġyn özüja jonma abaq

$\{147\}$ [18] heč sufat ${ }^{157}$ byla ne ki körsej ${ }^{158}$ köklerde joġartyn da ne ki jer-

\{148\} [19] de ašağartyn da ne ki suvlarda ašagaraq jerge.

\{149\} [20] Bašurmaġn alarġa da ${ }^{159}$ qulluq etmegin alarǵa ki menmen

\{150\} [21] Adonaj tenrij künülevčü tenri saġynuvču günexin atalarnyn

$\{151\} \quad[22] \quad\{\{$ catchword:\}\} jaman

\section{Page $117 \mathrm{r}^{0}$}

\{152\} [1] jaman ulanlar üstüne üčünčü dorġadejin ${ }^{160}$ da dörtünčü

\{153\} [2] dorgadejin ${ }^{161}$ dušmanlaryma ${ }^{162}$. ${ }^{16 a^{163}}$ qyluvčudur men

$\{154\}$ [3] šavagat Łmin dorlarga ${ }^{164}$ süvüvčülerǵa oxuma toramny da

\{155\} [4] saqlavčularga micvalarymny. לא. Antetmegin šeminden ${ }^{165}$

$\{156\}$ [5] Adonaj tenrijnin jalgangàa ki Łkönü etmesti ${ }^{166}$ Adonaj Łanyda

$\{157\}[6]$ kim $^{167}$ ki antetse šemind'an anyn muftqada. זכור.

$\{158\}$ [7] Saġynġyn ošol ${ }^{168}$ šabat künnü azizlikta tutma any.

\{159\} [8] ששת Alty künlerda išlegin da qylg̀yn bar išijni.

$\{160\}$ [9] ni. Da ${ }^{169}$ ol jedinči kün šabatty Łmaxtavuna Adonaj

\{161\} [10] tenrijnin ${ }^{170}$ qylmaġyn heč iš sen da uvluj da qyzyj quluj da

\footnotetext{
${ }^{156}$ JSul.III.o1: on sözlerni.

157 JSul.III.01: tirsin.

${ }^{158}$ JSul.III.01: this word is absent.

159 JSul.III.01: any.

${ }^{160}$ JSul.III.01: dor istine.

${ }^{161}$ JSul.III.01: dor istine

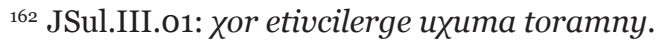

163 JSul.III.01: vale.

164 JSul.III.o1: minde dorlarga.

165 JSul.III.01: osol šeminden.

166 JSul.III.01: bos qojmasty.

167 JSul.III.o1: osol kimgede.

168 JSul.III.01: osol ol.

${ }^{169}$ JSul.III.01: vale.

${ }^{170}$ JSul.III.01: H-ga tenrine.
} 
\{162\} [11] qaravašyj da tuvaryj da garipijde ${ }^{171}$ ki šaharlaryjda. כי. Ki

$\{163\}$ [12] alty „künlernin ičind'a ${ }^{172}$ jaratty Adonaj ošol ol köklerni

$\{164\}$ [13] da ošol ol jerni ošol ol tengizni da ošol barča ne ${ }^{173} \mathrm{ki}$

$\{165\} \quad[14]$ alarda da เtynč etti ${ }^{174}$ ol jedinči künde anyn üčün

\{166\} [15] algyšlady Adonaj ošol ol šabat künnü da aziz etti any.

\{167\} [16] Syjlağyn ošol atajny da ošol anajny anyn üčün

\{168\} [17] uzaryrlar ${ }^{175}$ künlerij ${ }^{176}$ ol jer üstüne ki Adonaj tenrij ${ }^{177}$ beredi

\{169\} [18] saja. לא תרצח. Qaraqčylyq etmegin. לא תנאף. Niuf-

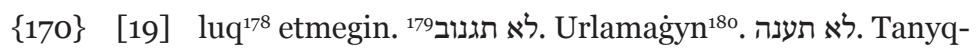

\{171\} [20] lyq čyqmaġyn Łdostuj üčünde ${ }^{7181}$ bolma jalgan tanyq. לא.

\{172\} [21] Suqlanmaġyn üvüne dostujnun suqlanmaġyn qatynyna dostuj-

\section{Page $117 \mathrm{~V}^{\mathrm{0}}$}

\{173\} [1] nun da quluna anyn da qaravašyna ${ }^{182}$ da ögüzüne da ešigi-

\{174\} [2] ne da Lbarysynada ne ki dostujnun ${ }^{7183}$. וכל. Da bar ol

\{175\} [3] ulus körerediler ošol ol avazlarny da ošol ol küsöv-

\{176\} [4] lerni da ošol ol šofar avazyn da ošol ol tavny tütün-

\{177\} [5] lenedoġanny ${ }^{184}$ \{dakördüulus\} da büreldiler ${ }^{185}$ da turdularjyraqtyn ${ }^{186}$.

$\{178\} \quad[6]$ Da ajttylar Mošege sözlegin sen birgemizge ${ }^{187}$ da tynlajyq ${ }^{188}$

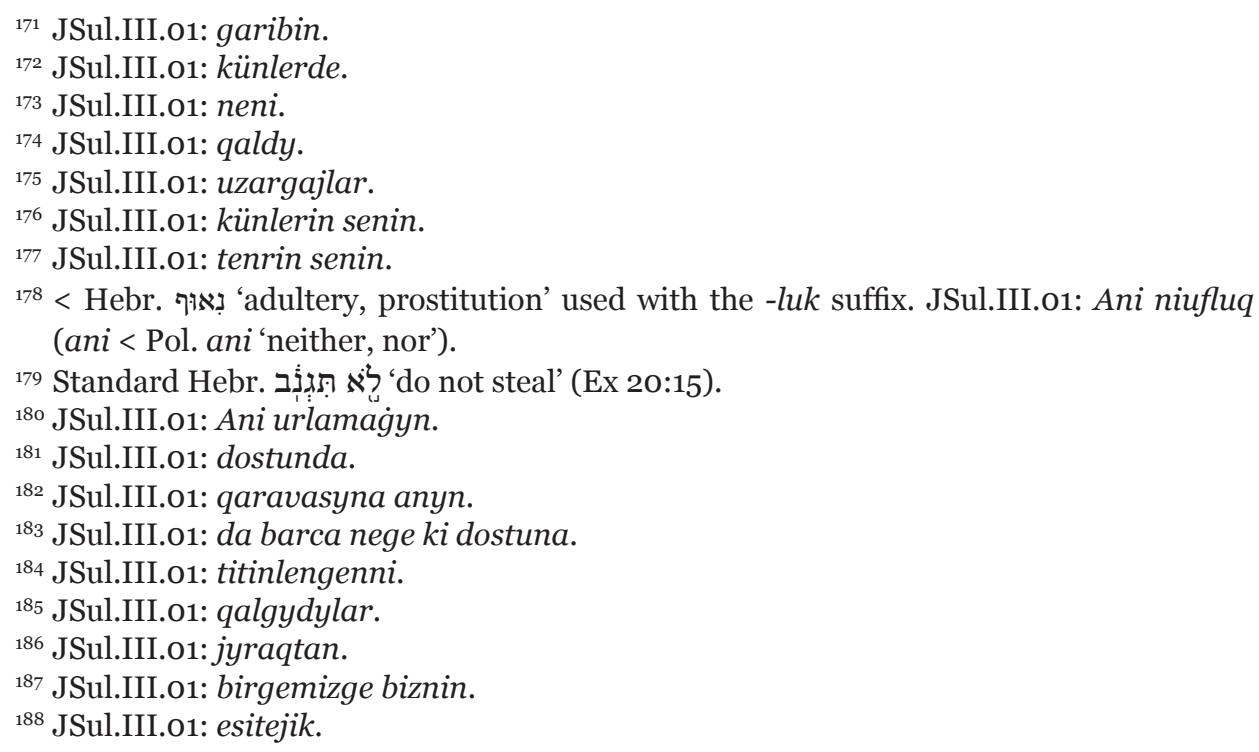


\{179\} [7] da sözlemesin Lbirgemizge tenri ${ }^{189}$ magat ölerbiz. ויאמר.

\{180\} [8] Da ajtty Moše ol ulusqa qorqmajyz ki čynyqtyrmaq ${ }^{190}$

$\{181\} \quad[9]$ üčün sizni $\left\llcorner q\right.$ orquvuna özünün ${ }^{191}$ keldi šexinasy ol tenrinin

\{182\} [10] da Lbolmaq üčün ${ }^{192}$ qorquvu anyn Łjüzlerijiz üstüne anyn

\{183\} [11] üčün ${ }^{193}$ ki jazyqly bolmaġyjsiz. ויעמד. Da turdu ol u-

\{184\} [12] lus jyraqtyn ${ }^{194}$ da Moše juvudu ol tumanga ki anda šexi-

\{185\} [13] nasy ol tenrinin. Da ajtty Adonaj Moše-

\{186\} [14] ge bulaj ajtqyn ulanlaryna ${ }^{195}$ Israelnin siz kördüjüz ki

\{187\} [15] Łnečik sözledim birgejizge ol köklerden körmedijiz heč

\{188\} [15] türsün ${ }^{196}$. לא. LAnyn üčün ${ }^{197}$ qylmajyz alnymda ${ }^{198}$ kümüš

\{189\} [16] abaqlar da altyn abaqlar qylmajyz özüjüzge. ${ }^{199}$

\subsection{Translation}

\section{Exodus 18:1-27}

\section{Page $113 \mathrm{~V}^{\mathrm{o}}$}

\{1\} [1] (1) And Jethro, the priest

\{2\} [2] of Midian, Moses' father-in-law, heard of all

\{3\} [3] that God had done for Moses, and for Israel his people,

\{4\} [4] that the Lord had brought Israel out of Egypt; (2) and

\{5\} [5] Jethro, Moses' father in law, took Zipporah, Moses' wife,

\{6\} [6] after he had sent her away, (3) and her two sons;

$\{7\}$ [7] of which the name of the one was Gershom; for he said, I have been alone

\{8\} [8] in a strange land: (4) and the name of the one ${ }^{200}$ was Eliezer, who said

\footnotetext{
${ }^{189}$ JSul.III.01: tenri birgemizge.

190 JSul.III.01: synamaq.

${ }^{191}$ JSul.III.O1: this fragment is absent.

192 JSul.III.o1: anyn ücün bolgaj.

193 JSul.III.o1: alnynyzda siznin.

194 JSul.III.o1: jyraqtan.

195 JSul.III.01: uvullaryna.

${ }^{196}$ JSul.III.o1: ol köklerden sözledim birgenizge siznin.

${ }^{197}$ JSul.III.O1: this fragment is absent.

${ }^{198}$ JSul.III.o1: birgeme menim.

${ }^{199}$ The last three verses of this chapter (i.e. Exodus 20:24-26) belong to the parashah Mishpatim.

${ }^{200}$ Instead of 'the other', probably a clerical error in the Karaim copy; cf. the translation in JSul.III.01.
} 
\{9\} [9] that "The God of my father was my help, and delivered

\{10\} [10] me from the sword of Pharaoh:" (5) and Jethro, father-in-law

\{11\} [11] of Moses, and his sons and his wife came unto Moses into the wilderness,

\{12\} [12] where he rested at the mount of God: (6) and

\{13\} [13] he said unto Moses by a messenger, I your father-in-law Jethro am come

\{14\} [14] unto you, and your wife, and her two sons with her. (7)

\{15\} [15] And Moses went out to meet his father-in-law, and bowed, and

\{16\} [16] kissed him; and they asked each other of their health; and

$\{17\}$ [17] they came into the tent. (8) And Moses told his father-in-law

\{18\} [18] all that the Lord had done unto Pharaoh and to the Egyptians

\{19\} [19] for Israel's sake, and all the torment that had come upon them by the way, $\{20\}[20]$ and how the wicked fought with them and how the Lord delivered them. (9)

\{21\} [21] And Jethro rejoiced for all the goodness which the Lord had done to Israel,

$\{22\}[22]\{\{$ catchword: $\}\}$ in that he had delivered

\section{Page $114 \mathrm{r}^{\circ}$}

\{23\} [1] in that he had delivered out of the hand of the Egyptians. (10) And Jethro said,

\{24\} [2] "Blessed be the Lord, who has delivered you out of the hand of the Egyptians, and

\{25\} [3] out of the hand of Pharaoh, who has delivered the people from under the reign

\{26\} [4] of the Egyptians. (11) Now I know that the Lord is greater

\{27\} [5] than all gods ${ }^{201}$ : for in the the way they plotted their works,

\{28\} [6] so did he pay them back." (12) And Jethro,

\{29\} [7] Moses' father-in-law, took a burnt offering and sacrifices for God: and Aaron came,

\{30\} [8] and all the elders of Israel, to eat bread with Moses' father-in-law

\{31\} [9] before God. (13) And it came to pass on the morrow,

\{32\} [10] that Moses sat to judge the people: and the people stood

\{33\} [11] before Moses from the morning unto the evening.

\{34\} [12] (14) And when Moses' father-in-law saw all that he

\{35\} [13] did to the people, he said, "What is this thing that you do

\{36\} [14] to the people? why do you alone sit to judge the people,

$\{37\}[15]$ and all the people stand due to the great number of the judgements

\{38\} [16] before you from morning unto evening?” (15) And Moses said

\{39\} [17] unto his father-in-law, "Because a part of ${ }^{202}$ the people come unto me to inquire of

${ }^{201}$ Literally: 'angels'.

${ }^{202}$ Uncertain translation, see our remark in the transcription. 
\{40\} [18] the words of God: (16) and so, when there is a word of judgement for them,

$\{41\}$ [19] they come unto me; and I judge between one and another, and

$\{42\}$ [20] between friends, and for those who inquire about the words of God

\{43\} [21] I do make them know the statutes of God, and

\section{Page $114 \mathrm{~V}^{0}$}

\{44\} [1] his teachings.” (17) And Moses' father-in-law

\{45\} [2] said unto him, "The thing that you do is not good. (18)

\{46\} [3] You will surely wear away, both you, and this people that is with you: for

$\{47\}$ [4] this thing is too heavy for you; you are not able to perform it yourself alone.

\{48\} [5] (19) Hearken now unto my voice, I will give you counsel, and

\{49\} [6] God's help shall be with you: Represent the people

\{50\} [7] before God, that you may bring the causes

\{51\} [8] unto God: (20) And you shall order them

$\{52\}$ [9] statutes and teachings, and shall make them know

$\{53\}[10]$ the way in which they must walk, and the work that they

\{54\} [11] must do. (21) Moreover, you shall watch to provide out of all the people brave

\{55\} [12] men, such as fear God, men of truth, hating stolen

$\{56\}$ [13] gain; and place such over them, to be the rulers of thousands, and rulers

\{57\} [14] of hundreds, rulers of fifties, and rulers of tens:

\{58\} [15] (22) and let them judge the people at all

\{59\} [16] times: and it shall be, that every great matter they shall bring unto you,

\{60\} [17] but every small matter they shall judge by their own: so shall it be easier

$\{61\}$ [18] for yourself, and they shall bear [the burden] with you. (23) If

\{62\} [19] you shall do this thing, and God command you so, then you shall be able

$\{63\}$ [20] to endure, and all this people shall also go to their place

$\{64\}$ [21] in peace. (24) So Moses hearkened to the voice of his father-in-law,

$\{65\}[22]\{\{$ catchword: $\}\}$ and did

\section{Page $115 \mathrm{r}^{0}$}

\{66\} [1] and did all that he had said. (25) And Moses chose brave

$\{67\}[2]$ men out of all Israel, and made them heads over the people,

$\{68\}$ [3] rulers of thousands, rulers of hundreds, rulers of

\{69\} [4] fifties, and rulers of tens. (26) And they judged

$\{70\}[5]$ the people at all times: they brought hard judgements ${ }^{203}$

\footnotetext{
${ }^{203}$ Literally: 'words'.
} 
\{71\} [6] unto Moses, but every small matter they

\{72\} [7] judged themselves. (27) And Moses let

\{73\} [8] his father-in-law depart; and he went his way into his own land....

\section{Exodus 19:1-25}

\{74\} [9] month, when the children of Israel were gone forth out of the land of Egypt,

$\{75\}[10]$ the same day came they into the wilderness of Sinai. (2) And

\{76\} [11] they had departed from Rephidim, and had come to the desert of Sinai, and had

\{77\} [12] camped in the wilderness; and there Israel camped before

\{78\} [13] the mount. (3) And Moses went up unto God,

\{79\} [14] and the Lord called unto him out of the mountain, saying, "Thus shall you say

$\{80\}[15]$ to the house of Jacob, and tell the children of Israel:

\{81\} [16] (4) 'You have seen what I did unto the Egyptians, and

\{82\} [17] how I bore you like on eagles' wings, and

\{83\} [18] brought you unto myself. (5) Now therefore, if

$\{84\}$ [19] you listen to my voice, and keep my covenant,

$\{85\}$ [20] then you shall be the chosen one unto me above all people: for mine is

\{86\} [21] all the earth; (6) And you shall be unto me a kingdom of priests,

\section{Page $115 \mathrm{~V}^{0}$}

\{87\} [1] and a holy kingdom'. These are the words which you shall speak unto the children

\{88\} [2] of Israel.” (7) And Moses came and called for the elders

\{89\} [3] of the people, and laid before their faces all these words

\{90\} [4] which the Lord conveyed through him. (8) And

\{91\} [5] all the people answered together, and said, "All that the Lord has spoken

\{92\} [6] we will do.” And Moses returned the words of the

\{93\} [7] people unto the Lord. (9) And the Lord said unto Moses,

\{94\} [8] "Lo, my Divine Presence will come unto you in a thick cloud, that

$\{95\} \quad[9]$ the people may hear when I speak with you, and

\{96\} [10] believe you forever." And Moses told the

\{97\} [11] words of the people unto the Lord. (10) And the Lord said

\{98\} [12] unto Moses, "Go unto the people, and sanctify them

\{99\} [13] today and tomorrow, and let them wash their clothes,

$\{100\}$ [14] (11) and be ready by the third day: for

\{101\} [15] the third day the Lord will come his Divine Presence down in the sight 
\{102\} [16] of all the people upon mount Sinai. (12)

\{103\} [17] And you shall set bounds unto the people round about, saying, 'Take heed

\{104\} [18] to yourselves, that you go not up into the mount, or touch the border of it:

\{105\} [19] whosoever touches the mount shall be put to death: (13)

\{106\} [20] And whosoever leaves the bounds there shall not a hand touch it, but

$\{107\}[21]$ if he will be close he shall surely be stoned, or

$\{108\}[22]\{\{$ catchword: $\}\}$ if

\section{Page $116 \mathrm{r}^{\circ}$}

\{109\} [1] he will be far, shot through with an arrow; whether it be man

\{110\} [2] or beast, it shall not live: when the trumpet made of

\{111\} [3] ram's horn sounds long, they shall come after standing up from their tents

\{112\} [4] up to the mount. (14) And Moses went down from the mount

\{113\} [5] unto the people, and sanctified the people; and they washed

\{114\} [6] their own clothes. (15) And he said unto the people,

$\{115\} \quad[7]$ "Be ready by the third day: come not near your wives."

\{116\} [8] (16) And it came to pass on the third day in the morning,

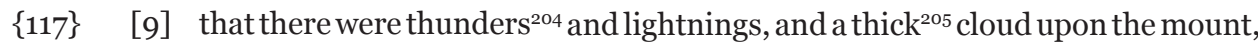

\{118\} [10] and the voice of the trumpet very loud; so that all the people trembled

\{119\} [11] who were in the camp. (17) And Moses brought forth the people

\{120\} [12] out of the camp before God; and they stood at the lower part

\{121\} [13] of the mount. (18) And mount Sinai was completely in smoke, because

\{122\} [14] the Lord's Divine Presence descended upon it in fire:

\{123\} [15] and the smoke of it ascended as the smoke of a furnace, and

\{124\} [16] the whole mount quaked greatly. (19) And when the voice of the trumpet

$\{125\}[17]$ sounded long, and grew louder and louder, Moses spoke,

$\{126\} \quad[18]$ and God answered ${ }^{206}$ him by a voice of strength that

\{127\} [19] the children of Israel shall hearken unto the words of Moses when

$\{128\}$ [20] the trumpet sounds long. (20) And the Divine Presence of Lord came down

\{129\} [21] upon mount Sinai, on the top of the mount: and the Lord called

\section{Page $116 \mathrm{~V}^{\mathrm{o}}$}

\{130\} [1] Moses up to the top of the mount; and Moses went up. (21) And

\{131\} [2] the Lord said unto Moses, "Go down, testify among the people, lest

\footnotetext{
204 Literally: 'noises'.

${ }^{205}$ Literally: 'strong'.

${ }^{206}$ Literally: 'repeated'.
} 
\{132\} [3] they break through the border unto the Lord to gaze, and perish

\{133\} [4] many of them. (22) And let the priests also, who come before

$\{134\} \quad[5]$ the Lord, sanctify themselves, lest the Lord break forth upon them."

\{135\} [6] (23) And Moses said unto the Lord, "The people cannot

\{136\} [7] come up to mount Sinai: for you charged us, saying,

\{137\} [8] 'Set bounds about the mount, and sanctify it."' (24) And the Lord said

\{138\} [9] unto him, "Go, get down, and then you shall come up, you, and Aaron with you:

\{139\} [10] but then, as I ordered you, let not the priests and

\{140\} [11] the people break through the border to come up before the Lord, lest

\{141\} [12] he break forth upon them." (25) And Moses went down unto the people,

\{142\} [13] and spoke unto them ...

\section{Exodus 20:1-23}

\{142\} [13] $\quad$... (1) And God spoke

\{143\} [14] all these words, saying, (2) "I am

\{144\} [15] the Lord your God, who has brought you out of the land of Egypt,

$\{145\} \quad[16]$ out of the power of slaves. (3) You shall have no other gods

\{146\} [17] before my power. (4) You shall not make unto yourself any graven image,

$\{147\} \quad[18]$ or any likeness of any thing that is in heaven above, or that is in the earth

\{148\} [19] beneath, or that is in the water under the earth: (5)

\{149\} [20] You shall not bow down yourself to them, nor serve them: for I

\{150\} [21] the Lord your God am a jealous God, remembering the iniquity of the fathers upon the

$\{151\} \quad[22] \quad\{\{$ catchword: $\}\}$ evil

\section{Page $117 \mathrm{r}^{\mathrm{O}}$}

\{152\} [1] evil children unto the third generation and fourth

\{153\} [2] generation of them that hate me; (6) and showing

\{154\} [3] mercy unto thousands of them that love reading my law, and

\{155\} [4] keep my commandments. (7) You shall not swear to the name

\{156\} [5] of the Lord your God falsely; for the Lord will not justify him

\{157\} [6] that swear to his name in vain. (8)

\{158\} [7] Remember the Sabbath day, to keep it holy.

\{159\} [8] (9) Six days shall you labour, and do all your work.

$\{160\}$ [9] (10) But the seventh day is the Sabbath for praising the Lord 
\{161\} [10] your God: in it you shall not do any work, you, nor your son, nor your daughter, your manservant, nor

\{162\} [11] your maidservant, nor your cattle, nor your stranger that is within your cities: (11) For

\{163\} [12] in six days the Lord made heaven

\{164\} [13] and earth, the sea, and all that

\{165\} [14] is in them, and rested the seventh day: therefore

\{166\} [15] the Lord blessed the Sabbath day, and hallowed it.

\{167\} [16] (12) Honor your father and your mother: that your

\{168\} [17] days may be long upon the land which the Lord your God gives

\{169\} [18] you. (13) You shall not kill. (14) You shall not commit

\{170\} [19] adultery. (15) You shall not steal. (16) You shall not bear

$\{171\} \quad[20]$ false witness against your neighbor. (17)

$\{172\}$ [21] You shall not covet your neighbour's ${ }^{207}$ house, you shall not covet your neighbour's wife,

\section{Page $117 \mathrm{~V}^{0}$}

\{173\} [1] nor his manservant, nor his maidservant, nor his ox, nor his donkey,

\{174\} [2] nor any thing that is your neighbour's. (18) And all the

$\{175\} \quad[3]$ people saw the thunderings, and the lightnings, ${ }^{208}$

\{176\} [4] and the noise of the trumpet, and the mountain smoking:

\{177\} [5] and when the people saw it, they moved, and stood far off. (19)

\{178\} [6] And they said unto Moses, "Speak you with us, and we will hear:

\{179\} [7] but let not God speak with us, lest we die." (20)

\{180\} [8] And Moses said unto the people, "Fear not: for to accustom

\{181\} [9] you to his fear God's Divine Presence has come

\{182\} [10] and that his fear may be before your faces,

\{183\} [11] that you sin not." (21) And the

\{184\} [12] people stood far off, and Moses drew near unto the thick darkness where

\{185\} [13] God's Divine Presence was. (22) And the Lord said unto Moses,

\{186\} [14] "Thus you shall say unto the children of Israel, 'You have seen that

\{187\} [15] I have talked with you from heaven and you have not seen any

\{188\} [16] figure." (23) Therefore you shall not make before me gods of silver,

$\{189\}[17]$ or gods of gold for yourselves.

${ }^{207}$ Literally: 'friend's', consequently in the whole sentence.

${ }^{208}$ Literally: 'the noises and the torches'. 


\section{Linguistic remarks}

\subsection{The $e>$ 'a shift}

From the above sample it is clear that the Torah translation originates from a period when the harmony shift was still an ongoing process. If we take, however, a closer look at the forms that exhibit the $e>$ ' $a$ change (see Table 1), i.e. the only element of the harmony shift that was reflected in writing, the following additional observations are valid.

The $e>$ ' $a$ change occurs in the following words:

\begin{tabular}{|c|c|c|c|c|}
\hline $\begin{array}{l}\text { Line } \\
\text { № }\end{array}$ & $\begin{array}{c}\text { In manuscript } \\
\text { III-73 }\end{array}$ & $\begin{array}{l}\text { In original } \\
\text { orthography }\end{array}$ & $\begin{array}{l}\text { The form prior } \\
\text { to harmony shift }\end{array}$ & $\begin{array}{l}\text { Present-day } \\
\text { form }\end{array}$ \\
\hline 4 & Micriǵan & מִצְרְידָין & Micriden & [Ḿićŕidań] \\
\hline 8 & jerd'a & "יִיְרְדָיא & jerde & [jerd'a] \\
\hline 14 & birgesińa & בִירְגְיסִינְיא & birgesine & [biǵaśińa] \\
\hline 18 & Micriǵa & מִצְרְיגזיא & Micrige & [Ḿiććriǵa] \\
\hline $25-6$ & erkitüvünd’an & אַרְכִיטיוּביוּנְדְין & erkitüvünden & [erkit'uvundań] \\
\hline 29 & tenriǵa & טַנְרִיגָיה & tenrige & [téńríiga] \\
\hline 32 & etḿa & אִטְמָיא & etme & [et'́na] \\
\hline 37 & köplügündan & כיוֹפְליוּגיוּנְדָדין & köplügünden & [ḱoṕĺuǵuńdań] \\
\hline 47 & send'an & סֵנְדָין & senden & [śeńdáné] \\
\hline 55 & tenridáan & טַנְרְידָין & tenriden & [téńríidań] \\
\hline 61 & üstüjdan & איוּסְטיוּיְיְָדָין & üstüjden & [üśtúujdań] \\
\hline 61 & birgeja & בִירְגְייָא & birgeje & [biŕǵeja] \\
\hline 73 & jerińa & יִיִרנְיא & jerine & [jeŕińa] \\
\hline 74 & jerind'an & יִיירנדְדָין & jerinden & [jeŕińdań] \\
\hline 95 & sözlegenimdá & סיוֹזְלֵיגינינימדָדָא & sözlegenimde & [śoźĺaǵańiḿd’a] \\
\hline 106 & čektáan & צַכְטָין & čekten & [č́éktáán] \\
\hline 111 & müvüzünd’an & מיוּביוּזיוּנְדָין & müvüzünden & [ḿuv́uźuńǵań] \\
\hline 115 & künlerǵa & כיוּנְלְירגָגיאה & künlerge & [ḱuńlááda] \\
\hline 134 & azizlensinĺar & 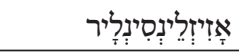 & azizlensinler & [aźiźlańśinińlar] \\
\hline 144 & jerind'an & ייִירְנָדין & jerinden & [jeŕińdań] \\
\hline 146 & özüja & איוֹזיוּיָיאָ & özüje & [öźuja] \\
\hline 154 & süvüvčülerǵa & 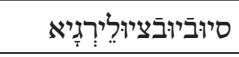 & süvüvčülerge & [śuv́uv́ćuláaŕga] \\
\hline
\end{tabular}




\begin{tabular}{|c|c|c|c|c|}
\hline $\begin{array}{c}\text { Line } \\
\text { № }\end{array}$ & $\begin{array}{c}\text { In manuscript } \\
\text { III-73 }\end{array}$ & $\begin{array}{c}\text { In original } \\
\text { orthography }\end{array}$ & $\begin{array}{l}\text { The form prior } \\
\text { to harmony shift }\end{array}$ & $\begin{array}{l}\text { Present-day } \\
\text { form }\end{array}$ \\
\hline 157 & šemind'an & שְמִינְדָין & šeminden & [šeḿińd’ań] \\
\hline 159 & künlerd'a & כיוּנְלִיְרָדָיא & künlerde & [ḱuńlaŕda] \\
\hline 163 & ičind'a & אִיצִנְדָיא & ičinde & [ičińda] \\
\hline
\end{tabular}

Table 1. Words that reveal the harmony shift in parashah Yitro (manuscript III-73)

These forms are clearly outnumbered by forms built according to the original vowel harmony. The ratio of the original $e$ to ' $a$ is 176 to 25, i.e. only $12.5 \%$ of the relevant word forms is shifted toward partial consonant harmony. Such pairs

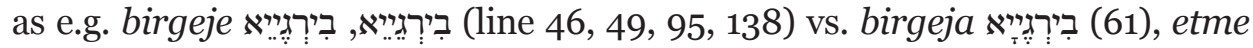

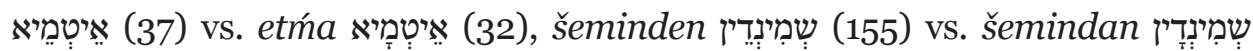
(157) prove that the innovative forms were alternating with the original ones.

The $e>$ ' $a$ change occurs only in final syllables, more precisely in suffixes and in the primary postposition köre attested as köra (or koŕa). ${ }^{209}$ There is no word longer than two syllables that would be fully assimilated with regard to this change. The $e>$ ' $a$ change operated irrespective of the accent, which is basically oxytonic in Karaim, given that in the manuscript we find negative verb forms, as e.g. körmejdilar כיוֹרְמֵיְדילְיר (Bo 18), in which the word final *-ler evolved into -lar whereas the accent falls on the syllable preceding the negative suffix -me (i.e. in this example on the initial syllable).

The $e>$ ' $a$ change operated less intensively after consonants that were already palatalized before the harmony shift stared to operate, $\mathrm{cf}$. the frequency of the occurrence of the segments /de/ i.e. [de] vs. /da/i.e. [da] which is 17 to 14 - juxtaposed with the ratio of /ge/i.e. [ǵe] to /ga/i.e. [ga] which is 45 to $4 .{ }^{210}$

These word forms show that the harmony shift operated most probably around the turn of the $17^{\text {th }}$ and $18^{\text {th }}$ centuries after the $\eta>j$ change (for an attempt to chronologize the latter change see JANKOWSKI 2014, in this volume). In certain idiolects or areas it must have ended before the 1720s; otherwise Simcha ben Chananiel could not have copied the fragments on folios $342 \mathrm{r}^{0}-385 \mathrm{v}^{0}$, which exhibit fully operational consonant harmony. Needless

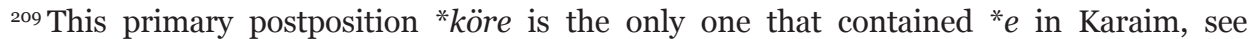
e.g. Németh (2011b: 103-104). It is not attested in the parashah Yitro, but it is used in the form köŕa כיוֹרָיא in other fragments, see e.g. parashah Bo, line 201.

${ }^{210}$ For a complex interpretation of this phenomenon see NÉmETH (2014b).
} 
to say, further investigation is needed to establish the time-frame of this phenomenon with greater accuracy.

\subsection{Rounded vs. unrounded vowel harmony}

Rounded vs. unrounded vowel harmony is fully developed in the text,

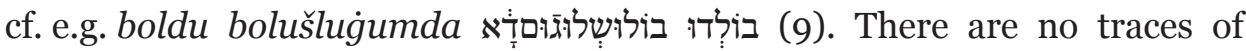
disharmonized forms like *boldy or *bolušlyg்ymda known from Crimean Karaim texts (even from the $19^{\text {th }} \mathrm{c}$.).

\section{5•3. The segment ${ }^{*} a j$}

There are no traces of the $a j>e j$ change ${ }^{211}$ we know from Modern North-West-

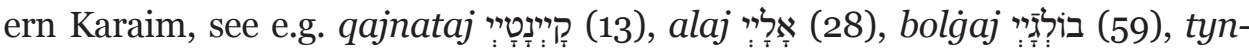
lasajyz טִנְלָסייזי (84), etc. in place of MNWKar. qajnatej, alej, bolgej, tynlasejyz, respectively. Instead, sporadically the segment $a j$ evolved into $y j$, which has hitherto been observed only in south-western Karaim texts (see Grzegorzewski 1916-1918: 258; Musaev 1964: 289; Németh 2011a: 49-50). The only example for the $a j>$ yj change in parashah Yitro is the word bolmagiyjsiz (183).

\subsection{The person markers}

The $1^{\text {st }}$ and $2^{\text {nd }}$ sg. person markers -men and -sen are attested in the manuscript in their original forms unaffected by the later process of -men $>-m y n$ -miń and -sen > -syn -śiń change, cf. e.g. etemen (ִילְ- (41), bildiremen

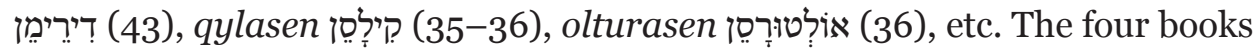
of Ketuvim exhibit the same feature (i.e. -men and -sen). The evolution of these person markers took place much later than the $e>$ ' $a$ change: we still encounter them in manuscripts from the late $19^{\text {th }}$ century, like e.g. in JSul.III. $31^{212}$ where we find forms like בִילַימֵין bilaḿeń 'I know' (22 ro), בירַיסֵין beraśeń 'you give' $\left(22 \mathrm{v}^{0}\right)$, etc.

\footnotetext{
${ }^{211}$ This change occurred only on morphologic boundaries and in suffixes.

${ }^{212}$ This item contains handwritten additions in north-western Karaim added to a printed siddur published in two parts in 1868 and 1872.
} 


\subsection{Slavonic loanwords}

Based on a brief preliminary analysis, we can say that the analysed Torah translation contains a certain number of Slavonic loanwords, which shows that the influence of the surrounding Slavonic languages must have been strong already at the time the Torah was translated (this corresponds with the idea that the harmony shift was triggered by the influence of Slavonic and Lithuanian phonotactics). This is especially true given the fact that the translations of Biblical texts were usually much more resistant to external influence - except Hebrew, of course. It might be therefore an interesting addition

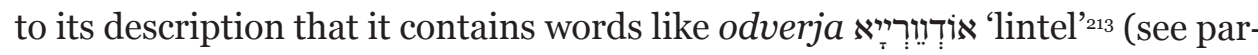

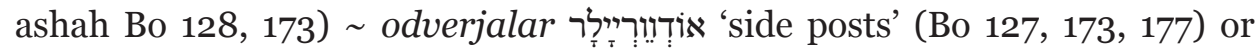

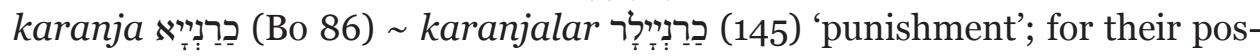
sible etymons cf. e.g. Russ. arch. одверье (sg.), одверья 'door-frame' (SRJa XII 265) and Russ. arch. каранъе (sg.), каранья 'punishment' (SRJa VII 73). The editors of KarRPS $(292,424)$ qualified both words as Polish, and so does Musaev (2003: 60) in respect to odverja, even though the Polish origin of both words is improbable for phonetic and semantic reasons; cf. the Polish metathetic form odrzwia 'door-frame' on the one hand, and, on the other hand, Pol. karanie 'punishing' with final $-e$ and slightly different meaning.

\section{Final remarks}

It is obvious that based on such a concise and preliminary comparison of the two Torah translations as it was presented above, the question of the relation of these manuscripts cannot be appropriately answered. On the one hand, there is a number of similarities that brings them closer (the lexicon is mostly the same with certain diverging tendencies, and there are only minor morphological and syntactic differences), but on the other hand, there are no major differences between them. ${ }^{214}$

\footnotetext{
${ }^{213}$ KarRPS (424) records the word only for south-western Karaim.

${ }^{214}$ For instance, in the translation copied by Jeshua-Josef Mordkowicz words with possessive suffixes are often additionally reinforced by the respective possessive pronouns, see e.g. künlerij (168) vs. künlerin senin (JSul.III.01) 'your days', tenrij (168) vs. tenrin senin (JSul.III.o1) 'your God', birgemizge (178) vs. birgemizge biznin 'with us', birgesina (14) vs. birgesine anyn 'with him' (JSul.III.o1), jüzlerijiz üstüne (182) vs. alnynyzda siznin (JSul.III.o1) 'before your faces', etc. Also, occasionally, different is the use of the
} 
And thus, although there are verses that are exactly the same in the two translations (cf. e.g. Ex 18:12, 19:11, etc.), in the vast majority of verses there are some minor differences, some of which are completely different (see e.g. Ex 19:13). It still seems therefore valid to say that there must have been a common translating tradition that shaped Bible translations (the same observation follows from Jankowski's (2009: 514) analysis), but the question remains where do the differences come from? Are they induced by the idiolect of the copyist or are they rather dialect-dependant? In other words, did the copyists interpret the translations by introducing preferential changes and amendments into the copied originals or were these changes essential for better understanding by those who read them?

Further research, above all, critical editions and comparative critical editions are needed to better understand the way Biblical texts were translated.

\section{Abbreviations}

arch. = archaic; Hebr. = Hebrew; Kipch. $=$ The language of the Codex Comanicus; $\mathbf{p l}$. = plural; sg. = singular; MNWKar. = Modern North-Western Karaim; Pol. = Polish; Russ. $=$ Russian; SWKar. $=$ south-western Karaim

\section{References}

von Gabain, Annemarie. 1959. Die Sprache des Codex Cumanicus. In: Deny, J., Grønbech, K., Scheel, H., Togan, Z.V. (eds.). Philologiae Turcicae Fundamenta. Wiesbaden, 46-72.

GrZegorzewski, Jan. 1916-1918. Caraimica. Język Łach-Karaitów. Rocznik Oryentalistyczny 1/2, 252-296.

JANkowski, Henryk. 2009. Translations of the Bible into Karaim. Religion Compass $3 / 4,502-523$.

Jankowski, Henryk. 2014. Two Karaim Religious Poems by Isaac b. Abraham Troki. [Karaite Archives 2].

genitive attributives in noun phrases, cf. nešer qanatlary üstüne (82) vs. qanatlary istine nešerlernin, the latter word order being shaped by Slavonic structural influence. 
KIzILov, Mikhail. 2009. The Karaites of Galicia. An Ethnoreligious Minority Among the Ashkenazim, the Turks, and the Slavs. 1772-1945. Leiden, Boston.

Kowalski, Tadeusz. 1929, Karaimische Texte im Dialekt von Troki (= Prace Komisji Orjentalistycznej Polskiej Akademji Umiejętności 11). Kraków.

KarRPS = BASKAKOv, N.A., ŠAPŠAL, S.M., ZAJončKovsKIJ, A. (eds.). 1974. Karaimskorussko-poĺskij slovaŕ. Moskva.

Musaev, Kenesbaj Musaevič. 1964. Grammatika karaimskogo jazyka. Fonetika i morfologija. Moskva.

MusaEv, Kenesbaj Musaevič. 2003. Sintaksis karaimskogo jazyka. Moskva.

NÉMETH, Michał. 2011a. Unknown Lutsk Karaim Letters in Hebrew Script $\left(19^{\text {th }}-20^{\text {th }}\right.$ Centuries). A critical edition (= Studia Turcologica Cracoviensia 12). Kraków.

NÉMETH, Michał. 2011b. Zwięzła gramatyka języka zachodniokaraimskiego z ćwiczeniami (= Prace Karaimoznawcze 1). Poznań.

NÉmEth, Michał. 2014a. Historical Phonology of Western Karaim: Alveolars and Front Labials in the South-western Dialect. Studia Linguistica Universitatis Iagellonicae Cracoviensis 131/3, 247-267.

Németh, Michał. 2014b. Historical Phonology of Western Karaim: The Evolution of Consonant Harmony in the North-western Dialect. [forthcoming in Studia Linguistica Universitatis Iagellonicae Cracoviensis 131/4].

Olach, Zsuzsanna. 2013. A Halich Karaim Translation of Hebrew Biblical Texts (= Turcologica 98). Wiesbaden.

SRJa VII = FiLIN F.P. (ed.). 1980. Slovaŕ russkogo jazyka XI-XVII vv. [Vol. VII: K-Kragujaŕ]. Moskva.

SRJa XII = Šmelev D.N. (ed.). 1987. Slovaŕ russkogojazyka XI-XVII vv. [Vol. XII: O-Oparnyj]. Moskva.

ZAJĄCZKOWSKI, Ananjasz. 1931. Krótki wykład gramatyki języka zachodnio-karaimskiego (narzecze tucko-halickie). Łuck.

\section{Acknowledgement}

This project was financed by the National Science Centre of Poland (Narodowe Centrum Nauki), grant number DEC-2011/03/D/HS2/o0618, and by The National Institute for Museums and Public Collection (Narodowy Instytut Muzealnictwa i Ochrony Zbiorów), grant number 00497/12/FPK/NIMOZ. 
Michał Németh (born 1980) is an assistant professor in the Institute of Linguistics at the Jagiellonian University in Kraków. He holds a PhD degree in Linguistics (2011) from the Jagiellonian University. His main interests lie in historical linguistics and etymology. He authored more than 30 articles and 3 books, among others Unknown Lutsk Karaim Letters in Hebrew script $\left(19^{\text {th }}-20^{\text {th }}\right.$ Centuries). A Critical Edition (Kraków, 2011) and Zwięzła gramatyka języka zachodniokaraimskiego z ćwiczeniami [= A Concise Grammar of Western Karaim with Exercises] (Poznań, 2011). His recent research concentrates on Karaim historical phonology and morphology, on critical editions of Karaim handwritten sources, and on the history of Hungarian lexicon. 


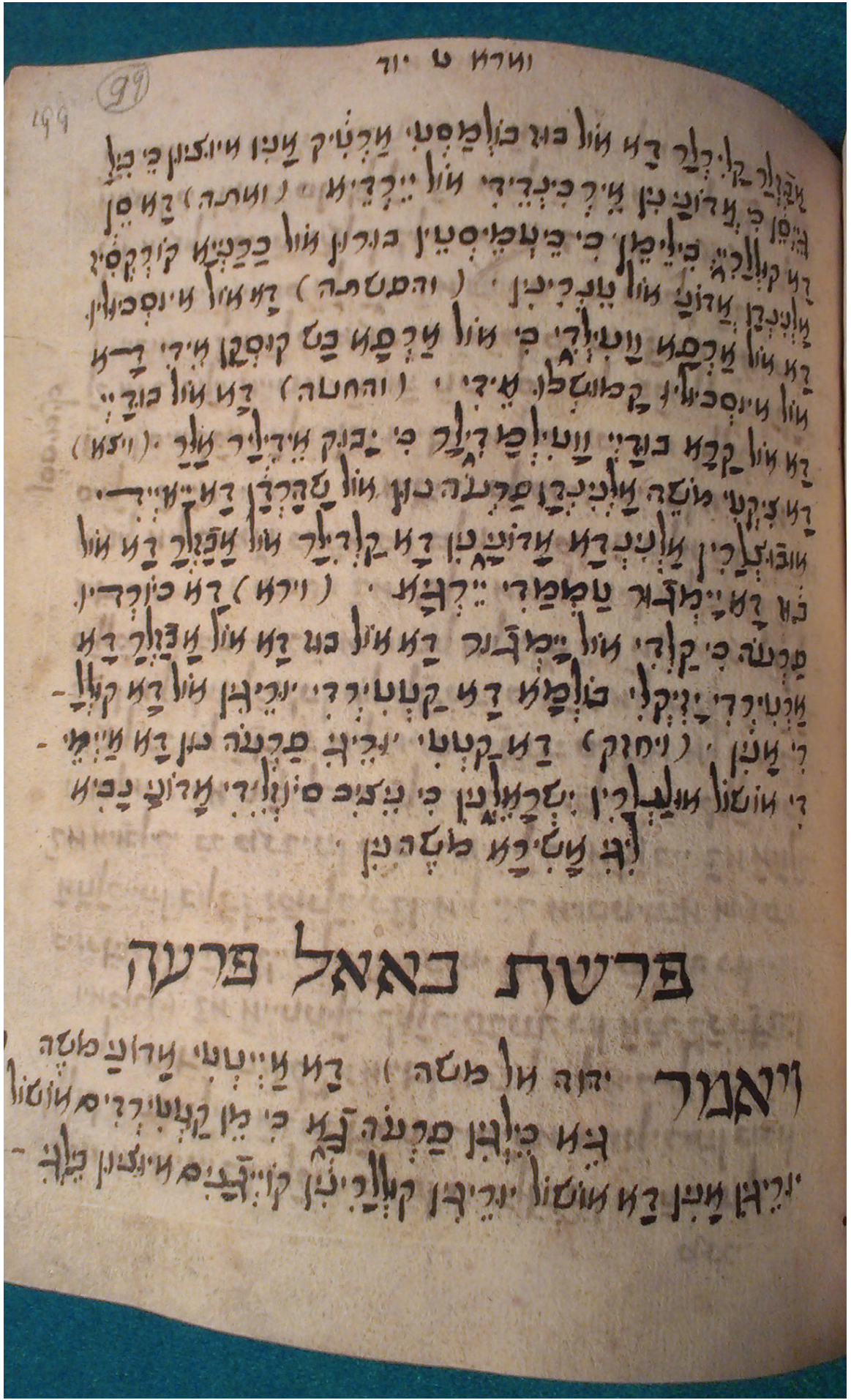

Facsimile of folio 99 recto 